\title{
Stability of constant steady states of a chemotaxis model
}

\author{
SZYMON CYGAN(D, GRZEGORZ KARCH(D), KRZYSZTOF KRAWCZYK(D) AND \\ HIROSHI WAKUID
}

Abstract. The Cauchy problem for the parabolic-elliptic Keller-Segel system in the whole $n$-dimensional space is studied. For this model, every constant $A \in \mathbb{R}$ is a stationary solution. The main goal of this work is to show that $A<1$ is a stable steady state while $A>1$ is unstable. Uniformly local Lebesgue spaces are used in order to deal with solutions that do not decay at spatial variable on the unbounded domain.

\section{Introduction}

There are several mathematical works on the chemotaxis model introduced by Keller and Segel [15]. Here, we refer the reader only to the monograph [25] and the reviews [3, 12] for a discussion of those mathematical results as well as for additional references. In this paper, we consider the following minimal parabolic-elliptic Keller-Segel system

$$
u_{t}-\Delta u+\nabla \cdot(u \nabla \psi)=0, \quad-\Delta \psi+\psi=u \text { for } t>0, \quad x \in \mathbb{R}^{n},
$$

where $u=u(t, x)$ denotes the density of cells and $\psi=\psi(t, x)$ is a concentration of chemoattractant. In these equations, all constant parameters are equal to one for simplicity of the exposition. System (1.1) is already studied in the whole space e.g. in the papers $[4-6,13,14,16,17,19]$, where several results either on a blow-up or on a large-time behavior of solutions have been obtained.

For each constant $A \in \mathbb{R}$, the couple $(u, \psi)=(A, A)$ is a stationary solution of system (1.1), and since the domain is unbounded, it does not belong to any Lebesgue $L^{p}$-space with $p \in[1, \infty)$. Thus, in Theorem 2.1 and in Sect. 3, we develop a mathematical theory concerning local-in-time solutions to the initial-value problem for system (1.1) in the uniformly local Lebesgue spaces $L_{\text {uloc }}^{p}\left(\mathbb{R}^{n}\right)$. Then, we consider a constant stationary solution $(u, \psi)=(A, A)$ with $A \in[0,1)$, and we show in Theorem 2.3 that a small $L^{p}$-perturbation of such an initial datum gives a global-in-time solution which converges toward $(A, A)$ as $t \rightarrow \infty$. On the other hand, we prove in Theorem 2.4 that the constant solution is unstable in the Lyapunov sense if $A>1$.

Mathematics Subject Classification: 35B35, 35B40, 35K15, 35K55, 35K92, 92C17

Keywords: Parabolic-elliptic Keller-Segel system, Constant steady states, Stability of solutions. This paper was invented and written online during the 2020 lockdown. 
A stability of constant solutions of chemotaxis models has been already studied in bounded domains. For example, the paper [10] describes dynamics near an unstable constant solution to the classical parabolic-parabolic Keller-Segel model in a bounded domain, and obtained results are interpreted as an early pattern formation. Another work [23] is devoted to the system

$$
u_{t}-\Delta u+\nabla \cdot(u \nabla \psi)=0, \quad-\Delta \psi+\mu=u, \quad \mu \equiv \frac{1}{\left|B_{R}(0)\right|} \int_{B_{R}(0)} u \mathrm{~d} x,
$$

in the ball of radius $R>0$ with Neumann boundary condition. Here, constants are also stationary solutions, and it is shown in the work [23] that there exists a critical number $m_{c}$ such that at mass levels above $m_{c}$ the constant steady states are extremely unstable and blow-up can occur. On the other hand, for $m<m_{c}$ there exist infinitely many radial solutions with a mass equal to $m$.

After this paper was deposed in arXiv and submitted for publication, we have found a recent paper [22] on local and global-in-time solutions to model (1.1) in a subspace of $L_{\mathrm{uloc}}^{p}$ defined below in Remark 3.4.

\section{Notation}

The usual norm of the Lebesgue space $L^{p}\left(\mathbb{R}^{n}\right)$ with respect to the spatial variable is denoted by $\|\cdot\|_{p}$ for all $p \in[1, \infty]$. In the following, we use also the uniformly local Lebesgue space $L_{\text {uloc }}^{p}\left(\mathbb{R}^{n}\right)$ with the norm $\|\cdot\|_{p \text {, uloc }}$ defined below by formula (2.3). Any other norm in a Banach space $Y$ is denoted by $\|\cdot\|_{Y}$. The letter $C$ corresponds to a generic constant (always independent of $t$ and $x$ ) which may vary from line to line. We write $C=C(\alpha, \beta, \gamma, \ldots)$ when we want to emphasize the dependence of $C$ on parameters $\alpha, \beta, \gamma, \ldots$. We use standard definition of Fourier transform $\widehat{f}(\xi)=$ $(2 \pi)^{-n / 2} \int_{\mathbb{R}^{n}} e^{-i \xi \cdot x} f(x) \mathrm{d} x$.

\section{Results and comments}

Our goal is to study properties of solutions to the Cauchy problem for the simplified parabolic-elliptic Keller-Segel model of chemotaxis

$$
\begin{cases}u_{t}-\Delta u+\nabla \cdot(u \nabla \psi)=0, & t>0, x \in \mathbb{R}^{n}, \\ -\Delta \psi+\psi=u, & t>0, x \in \mathbb{R}^{n}, \\ u(0, x)=u_{0}(x), & x \in \mathbb{R}^{n},\end{cases}
$$

with $n \geq 1$. We solve the second equation with respect to $\psi$ to obtain $\psi=K * u$, where $K$ is the Bessel function (see Lemma 3.6), which reduces problem (2.1) to the following one

$$
\begin{cases}u_{t}-\Delta u+\nabla \cdot(u \nabla K * u)=0, & t>0, \quad x \in \mathbb{R}^{n}, \\ u(0, x)=u_{0}(x), & x \in \mathbb{R}^{n}\end{cases}
$$


We begin by a result on an existence of local-in-time solutions to problem (2.2) in the uniformly local Lebesgue spaces

$$
L_{\mathrm{uloc}}^{p}\left(\mathbb{R}^{n}\right) \equiv\left\{f \in L_{\mathrm{loc}}^{p}\left(\mathbb{R}^{n}\right):\|f\|_{p, \mathrm{uloc}} \equiv \sup _{x \in \mathbb{R}^{n}}\left(\int_{B_{1}(x)}|f(y)|^{p} \mathrm{~d} y\right)^{1 / p}<+\infty\right\}
$$

for $p \in[1, \infty)$ and $L_{\mathrm{uloc}}^{\infty}\left(\mathbb{R}^{n}\right)=L^{\infty}\left(\mathbb{R}^{n}\right)$.

Theorem 2.1. For each $p$ satisfying

$$
\begin{gathered}
p \in[1, \infty] \text { if } n=1, \\
p \in\left[\frac{3}{2}, \infty\right] \text { if } n=2, \\
p \in\left(\frac{n}{2}, \infty\right] \text { if } n \geq 3,
\end{gathered}
$$

and every $u_{0} \in L_{\mathrm{uloc}}^{p}\left(\mathbb{R}^{n}\right)$, there exists $T>0$ and a unique mild solution

$$
u \in L^{\infty}\left([0, T) ; L_{\mathrm{uloc}}^{p}\left(\mathbb{R}^{n}\right)\right) \cap C\left((0, T) ; L_{\mathrm{uloc}}^{p}\left(\mathbb{R}^{n}\right)\right)
$$

of problem (2.2). Moreover, if $u_{0} \geq 0$, then $u(t, x) \geq 0$ almost everywhere in $[0, T) \times$ $\mathbb{R}^{n}$.

The more-or-less standard proof of Theorem 2.1 is based on the Banach contraction principle applied to an integral representation of solutions to problem (2.2) (see Sect. 3 for more details). In this work, we do not discuss regularity of solutions from Theorem 2.1 and we refer the reader to the work [22, Thm. 1.1] where more regular solutions have been constructed.

For $n=2$, one could expect that Theorem 2.1 holds true for all $p>1$. The extra condition $p \geq 3 / 2$ is introduced because the proof of this theorem is based on Lemma 3.9 where we require from the intersection (3.5) to be nonempty. This restriction on $p$ is not needed in the paper [22] where the solution is constructed in a smaller space, see also the reasoning from the proof of Theorem 2.3.

Now, we formulate a simple consequence of Theorem 2.1 in the case when an initial condition is an $L^{p}$-perturbation of a constant $A \in \mathbb{R}$.

Corollary 2.2. Let $p$ satisfy conditions (2.4). For every $A \in \mathbb{R}$ and every $v_{0} \in L^{p}\left(\mathbb{R}^{n}\right)$ there exists a unique local-in-time mild solution $u=u(t, x)$ of problem (2.2)(as stated in Theorem 2.1) corresponding to the initial datum $u_{0}=A+v_{0} \in L_{\mathrm{uloc}}^{p}\left(\mathbb{R}^{n}\right)$. This solution satisfies $u-A \in C\left([0, T) ; L^{p}\left(\mathbb{R}^{n}\right)\right)$.

This corollary is an immediate consequence of the uniqueness of solutions established in Theorem 2.1 combined with the uniqueness result of solutions to the perturbed problem considered in Proposition 5.1.

Next, we show that one can construct global-in-time solutions around each constant solution $A \in[0,1)$. 
Theorem 2.3. Let $A \in[0,1)$ and

$$
p=1 \text { if } n=1 \text { or } p \in\left(\frac{n}{2}, n\right] \text { if } n \geq 2 \text {. }
$$

Fix $q \in(n, 2 p]$. There exists $\varepsilon>0$ such that for every $v_{0} \in L^{p}\left(\mathbb{R}^{n}\right)$ with $\left\|v_{0}\right\|_{p}<\varepsilon$, problem (2.2) with the initial condition $u_{0}=A+v_{0}$ has a unique global-in-time mild solution $u(t, x)$ satisfying $u-A \in C\left([0, \infty) ; L^{p}\left(\mathbb{R}^{n}\right)\right)$ and

$$
\|u(t)-A\|_{p}+t^{\frac{n}{2}\left(\frac{1}{p}-\frac{1}{q}\right)}\|u(t)-A\|_{q} \leq C\left\|u_{0}-A\right\|_{p}
$$

for a constant $C>0$ and all $t>0$.

The estimate from Theorem 2.3 can be interpreted as a stability of each constant solution $A \in[0,1)$ in $L^{p}\left(\mathbb{R}^{n}\right)$ and asymptotic stability in $L^{q}\left(\mathbb{R}^{n}\right)$.

The smallness assumption imposed on initial conditions in Theorem 2.3 seems to be necessary. This is clear in the case $A=0$, where sufficiently large initial data lead to solutions which blow-up in finite time, see e.g. $[4,6,14,16]$ for blow-up results for solutions of system (1.1) considered in the whole space.

Next, we deal with $A>1$ which appears to be the unstable constant stationary solution.

Theorem 2.4. The constant stationary solution $A>1$ of problem (2.2) is not stable in the Lyapunov sense under small perturbations from $L^{p}\left(\mathbb{R}^{n}\right)$ for each $p$ satisfying condition (2.4) except $p=1$ and $p=\infty$.

In this theorem, we do not claim that solutions corresponding to $L^{p}$-perturbations of $A>1$ are global-in-time. We show only that if they are global-in-time, then they cannot be stable.

To conclude this section, we notice that a constant $A<0$ is linearly stable which we comment in Remark 4.8. The proof of nonlinear stability of this constant can be obtained by the method used in the proof of Theorem 2.3. We add some comments on the linear stability of the constant solution $A=1$ in Remark 4.9.

\section{Local-in-time solutions in uniformly local Lebesgue spaces}

We find solutions to problem (2.2) via its formulation in the integral form

$$
u(t)=e^{t \Delta} u_{0}-\int_{0}^{t} \nabla e^{(t-s) \Delta} \cdot(u(s) \nabla K * u(s)) \mathrm{d} s
$$

with the heat semigroup given by the formula

$$
\left(e^{t \Delta} f\right)(x) \equiv(4 \pi t)^{-\frac{n}{2}} \int_{\mathbb{R}^{n}} e^{-\frac{|x-y|^{2}}{4 t}} f(y) \mathrm{d} y .
$$

This construction requires auxiliary results which we are going to gather and prove below. Then, Theorem 2.1 is proved at the end of this section. 
Remark 3.1. Integral equation (3.1) is equivalent (for sufficiently regular solutions) to initial value problem (2.1) because of the relation

$$
\nabla e^{t \Delta} v_{0}=e^{t \Delta} \nabla v_{0}
$$

which can be justified by applying Fourier transform. Notice that relation (3.2) is usually not valid in a bounded domain for a heat semigroup with a suitable boundary condition.

Next, we recall properties of the heat semigroup $\left\{e^{t \Delta}\right\}_{t \geq 0}$ acting on the $L_{\mathrm{uloc}}^{p}$-spaces.

Proposition 3.2. [2,18] For all $1 \leq q \leq p \leq \infty, k \in \mathbb{Z}_{+}$and $\alpha \in \mathbb{Z}_{+}^{n}$ there exist numbers $C=C(n, p, q, k, \alpha)>0$ such that

$$
\left\|\partial_{t}^{k} \partial_{x}^{\alpha} e^{t \Delta} f\right\|_{p, \text { uloc }} \leq C t^{-k-\frac{|\alpha|}{2}}\left(1+t^{-\frac{n}{2}\left(\frac{1}{q}-\frac{1}{p}\right)}\right)\|f\|_{q, \mathrm{uloc}}
$$

for every $f \in L_{\mathrm{uloc}}^{p}\left(\mathbb{R}^{n}\right)$ and all $t>0$. In particular, when $p=q$ and $1 \leq p \leq \infty$, it holds that

$$
\left\|e^{t \Delta} f\right\|_{p, \text { uloc }} \leq\|f\|_{p, \text { uloc }} \text { for all } t \geq 0 \text {. }
$$

Remark 3.3. Proposition 3.2 generalizes the following well-known estimates of the heat semigroup acting on the Lebesgue space $L^{p}\left(\mathbb{R}^{n}\right)$

$$
\left\|\partial_{t}^{k} \partial_{x}^{\alpha} e^{t \Delta} f\right\|_{p} \leq C t^{-\frac{n}{2}\left(\frac{1}{q}-\frac{1}{p}\right)-k-\frac{|\alpha|}{2}}\|f\|_{q} \text { for all } t>0 .
$$

Remark 3.4. It follows from estimates (3.3) applied with $k=0, \alpha=0$ and $p=q$ that $e^{t \Delta} f \in L^{\infty}\left((0, \infty) ; L_{\mathrm{uloc}}^{p}\left(\mathbb{R}^{n}\right)\right)$ for each $f \in L_{\mathrm{uloc}}^{p}\left(\mathbb{R}^{n}\right)$ and, in general, this mapping in not continuous in time. This continuity holds true in the smaller space

$$
\mathscr{L}_{\mathrm{uloc}}^{p}\left(\mathbb{R}^{n}\right)=\overline{B U C\left(\mathbb{R}^{n}\right)}\|\cdot\|_{p, \text { uloc }},
$$

where $B U C\left(\mathbb{R}^{n}\right)$ is a space of all bounded uniformly continuous functions on $\mathbb{R}^{n}$. In fact, the following statements are equivalent:

1. $f \in \mathscr{L}_{\text {uloc }}^{p}\left(\mathbb{R}^{n}\right)$.

2. $\|f(\cdot+h)-f\|_{p, \text { uloc }} \rightarrow 0$ as $|h| \rightarrow 0$.

3. $\left\|e^{t \Delta} f-f\right\|_{p \text {,uloc }} \rightarrow 0$ as $t \rightarrow 0$.

We refer the reader to [18, Proposition 2.2] for the proof of these properties.

Remark 3.5. There is an alternative definition of the norm in the uniformly local $L^{p}$ spaces

$$
\|f\|_{p, \text { uloc }, \rho} \equiv \sup _{x \in \mathbb{R}^{n}}\left(\int_{B_{\rho}(x)}|f(y)|^{p} \mathrm{~d} y\right)^{1 / p}
$$

for each $\rho>0$. However, by a simple scaling property, we can show that all these norms are in fact equivalent. 
Let us also recall properties of the Bessel kernel, which are systematically used in this work.

Lemma 3.6. Denote by $\psi \in \mathscr{S}^{\prime}\left(\mathbb{R}^{n}\right)$ a solution of the equation $-\Delta \psi+\psi=u$ for some $u \in \mathscr{S}^{\prime}\left(\mathbb{R}^{n}\right)$ (the space of tempered distributions). The following statements hold true.

1. $\psi=K * u$, where $\widehat{K}(\xi)=\frac{1}{1+|\xi|^{2}}$.

2. For $n=1, K(x)=\frac{1}{2} e^{-|x|}$.

3. For $n \geq 2, K \in L^{1}\left(\mathbb{R}^{n}\right) \cap L^{p}\left(\mathbb{R}^{n}\right)$ for each $p \in\left[1, \frac{n}{n-2}\right)$ and $\nabla K \in L^{1}\left(\mathbb{R}^{n}\right) \cap$ $L^{q}\left(\mathbb{R}^{n}\right)$ for each $q \in\left[1, \frac{n}{n-1}\right)$.

4. $|\nabla K(x)|=\left|K^{\prime}(|x|)\right|=-K^{\prime}(|x|)$ is radially symmetric and decreasing in $|x|$.

Sketch of the proof. Property 1 is well known. Item 2 can be obtained by a direct calculation. For the proofs of properties 3 and 4 we refer the reader to [1, Sec. 1.2.5.]. In particular, in order to show property 4, we recall that $K(x)=(2 \pi)^{-\frac{n}{2}}|x|^{-\frac{n-2}{2}} K_{\frac{n-2}{2}}(|x|)$, where $K_{v}=K_{v}(r)\left(r>0, v>-\frac{1}{2}\right)$ stands for the modified Bessel function of the third kind, that is

$$
K_{\nu}(r)=\frac{\pi^{\frac{1}{2}}}{2^{\frac{1}{2}} \Gamma\left(\nu+\frac{1}{2}\right)} r^{-\frac{1}{2}} \mathrm{e}^{-r} \int_{0}^{\infty} s^{\nu-\frac{1}{2}}\left(1+\frac{s}{2 r}\right)^{\nu-\frac{1}{2}} \mathrm{e}^{-s} \mathrm{~d} s,
$$

see e.g. [1, formula (1.2.25), p.12]. In particular, we observe that

$$
K^{\prime}(r)=-\frac{1}{4 \pi}\left(\frac{2}{\pi}\right)^{\frac{1}{2}} r^{-\frac{n-2}{2}} K_{\frac{n}{2}}(r)
$$

Since $K_{\frac{n}{2}}(|x|)>0$ and $|x|^{-\frac{n-2}{2}}$ decreases in $|x|$, the function $-|x|^{-\frac{n-2}{2}} K_{\frac{n}{2}}(|x|)<0$ increases in $|x|$. Therefore, $|\nabla K(x)|=\left|K^{\prime}(|x|)\right|=-K^{\prime}(|x|)$ is radially symmetric and decreasing in $|x|$.

Our goal is to estimate the nonlinear term on the right-hand side of equation (3.1). We begin with the following elementary lemma.

Lemma 3.7. For $f \in L_{\mathrm{uloc}}^{p}\left(\mathbb{R}^{n}\right)$, it holds that

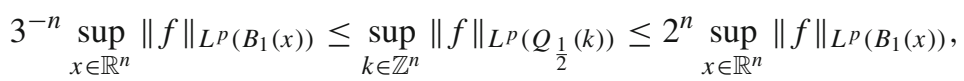

where $Q_{r}(k) \equiv\left\{\left(x_{1}, \cdots, x_{n}\right) \in \mathbb{R}^{n}: \max _{1 \leq j \leq n}\left|x_{j}-k_{j}\right| \leq r\right\}$ for $k=\left(k_{1}, \cdots, k_{n}\right) \in$ $\mathbb{Z}^{n}$ and $r>0$.

Proof. Obviously, it holds that $\mathbb{R}^{n}=\bigcup_{k \in \mathbb{Z}^{n}} Q_{1 / 2}(k)$ with $\left|Q_{1 / 2}(k) \cap Q_{1 / 2}(\ell)\right|=0$ for $k \neq \ell$. Thus, for $x \in \mathbb{R}^{n}$, there exists $k^{0} \in \mathbb{Z}^{n}$ such that $x \in Q_{1 / 2}\left(k^{0}\right)$. Since $x \in$ $Q_{1 / 2}\left(k^{0}\right)$, there exist lattice points $\left\{k^{\ell}\right\}_{\ell=1}^{3^{n}-1} \subset \mathbb{Z}^{n}$ such that $B_{1}(x) \subset \cup_{\ell=0}^{3^{n}-1} Q_{1 / 2}\left(k^{\ell}\right)$. Indeed, the set

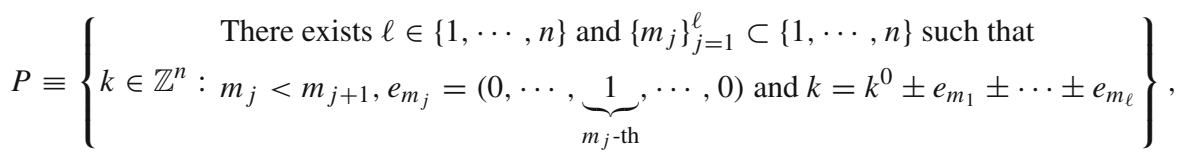


consists of $\left(3^{n}-1\right)$ points in $\mathbb{Z}^{n}$. Therefore, the number of elements of the set $\left\{k^{0}\right\} \cup P$ is $3^{n}$, and thus, we have

$$
\left(\int_{B_{1}(x)}|f(y)|^{p} \mathrm{~d} y\right)^{\frac{1}{p}} \leq \sum_{j=0}^{3^{n}-1}\left(\int_{Q_{\frac{1}{2}}\left(k^{j}\right)}|f(y)|^{p} \mathrm{~d} y\right)^{\frac{1}{p}} \leq 3^{n} \sup _{k \in \mathbb{Z}^{n}}\left(\int_{Q_{\frac{1}{2}}(k)}|f(y)|^{p} \mathrm{~d} y\right)^{\frac{1}{p}} .
$$

Hence we obtain the first part of our claim. On the other hand, it holds that $Q_{1 / 2}(k) \subset$ $\bigcup_{j=1}^{2^{n}} B_{1}\left(x^{j}\right)$, where $\left\{x^{j}\right\}_{j=1}^{2^{n}} \subset \mathbb{R}^{n}$ with $x_{\ell}^{j}=k_{\ell} \pm \frac{1}{2}$ for all $\ell=1, \cdots, n$ and $j=1, \cdots, 2^{n}$. Thus, we observe

$$
\left(\int_{Q_{\frac{1}{2}}(k)}|f(y)|^{p} \mathrm{~d} y\right)^{\frac{1}{p}} \leq \sum_{j=1}^{2^{n}}\left(\int_{B_{1}\left(x^{j}\right)}|f(y)|^{p} \mathrm{~d} y\right)^{\frac{1}{p}} \leq 2^{n} \sup _{x \in \mathbb{R}^{n}}\left(\int_{B_{1}(x)}|f(y)|^{p} \mathrm{~d} y\right)^{\frac{1}{p}} .
$$

Now, we prove a Young-type inequality in the space $L_{\mathrm{uloc}}^{p}\left(\mathbb{R}^{n}\right)$.

Proposition 3.8. Let $1+\frac{1}{p}=\frac{1}{q}+\frac{1}{r}$ with $1 \leq q \leq p \leq \infty$ and $1 \leq r<\frac{n}{n-1}$. Then, there exists a number $C=C(n)>0$ such that

$$
\|\nabla K * f\|_{p, \text { uloc }} \leq C\left(\|\nabla K\|_{1}+\|\nabla K\|_{r}\right)\|f\|_{q, \text { uloc }}
$$

Proof. This lemma has been proved in [18, Theorem 3.1] in the case of an arbitrary bounded and integrable function $K$. Since the Bessel kernel is not bounded for $n \geq 2$, we revise those arguments in our setting. Using the decomposition $\mathbb{R}^{n}=\bigcup_{k \in \mathbb{Z}^{n}} Q_{1 / 2}(k)$ and

$$
\nabla K(x)=\sum_{k \in \mathbb{Z}^{n}} \chi_{Q_{\frac{1}{2}}(k)}(x) \nabla K(x), \quad f(x)=\sum_{k \in \mathbb{Z}^{n}} \chi_{Q_{\frac{1}{2}}(k)}(x) f(x) \quad \text { a.e. } x \in \mathbb{R}^{n},
$$

we observe

$$
\begin{aligned}
\|\nabla K * f\|_{L^{p}\left(Q_{\frac{1}{2}}(k)\right)} & =\left\|\left(\sum_{k^{\prime} \in \mathbb{Z}^{n}} \chi_{Q_{\frac{1}{2}}\left(k^{\prime}\right)} \nabla K\right) *\left(\sum_{k^{\prime \prime} \in \mathbb{Z}^{n}} \chi_{Q_{\frac{1}{2}}\left(k^{\prime \prime}\right)} f\right)\right\|_{L^{p}\left(Q_{\frac{1}{2}}(k)\right)} \\
& =\left\|\sum_{k^{\prime}, k^{\prime \prime} \in \mathbb{Z}^{n}}\left(\chi_{Q_{\frac{1}{2}}\left(k^{\prime}\right)} \nabla K\right) *\left(\chi_{Q_{\frac{1}{2}}\left(k^{\prime \prime}\right)} f\right)\right\|_{L^{p}\left(Q_{\frac{1}{2}}(k)\right)} \\
& \leq \sum_{k^{\prime}, k^{\prime \prime} \in \mathbb{Z}^{n}}\left\|\left(\chi_{Q_{\frac{1}{2}}\left(k^{\prime}\right)} \nabla K\right) *\left(\chi_{Q_{\frac{1}{2}}\left(k^{\prime \prime}\right)} f\right)\right\|_{L^{p}\left(Q_{\frac{1}{2}}(k)\right)} .
\end{aligned}
$$

The well-known fact that supp $f_{1} * f_{2} \subset \operatorname{supp} f_{1}+\operatorname{supp} f_{2}$ implies that the support of the function $\left(\chi_{Q_{1 / 2}\left(k^{\prime}\right)} \nabla K\right) *\left(\chi_{Q_{1 / 2}\left(k^{\prime \prime}\right)} f\right)$ lies in the cube $Q_{1}\left(k^{\prime}+k^{\prime \prime}\right)$. Thus for 
$1 \leq p, q, r \leq \infty$ with $1+\frac{1}{p}=\frac{1}{q}+\frac{1}{r}$, the Young inequality shows that

$$
\begin{aligned}
& \sum_{k^{\prime}, k^{\prime \prime} \in \mathbb{Z}^{n}}\left\|\left(\chi_{Q_{\frac{1}{2}}\left(k^{\prime}\right)} \nabla K\right) *\left(\chi_{Q_{\frac{1}{2}}\left(k^{\prime \prime}\right)} f\right)\right\|_{L^{p}\left(Q_{\frac{1}{2}}(k) \cap Q_{1}\left(k^{\prime}+k^{\prime \prime}\right)\right)} \\
& \leq \sum_{k^{\prime}, k^{\prime \prime} \in \mathbb{Z}^{n}}\left\|\left(\chi_{Q_{\frac{1}{2}}\left(k^{\prime}\right)} \nabla K\right) *\left(\chi_{Q_{\frac{1}{2}}\left(k^{\prime \prime}\right)} f\right)\right\|_{L^{p}\left(Q_{\frac{1}{2}}(k) \cap Q_{1}\left(k^{\prime}+k^{\prime \prime}\right)\right)} \\
& \max _{1 \leq j \leq n}\left|k_{j}^{\prime}+k_{j}^{\prime \prime}-k_{j}\right| \leq 1 \\
& \leq \sum_{\substack{k^{\prime}, k^{\prime \prime} \in \mathbb{Z}^{n} \\
\max \left|k_{j}^{\prime}+k_{j}^{\prime \prime}-k_{j}\right| \leq 1}}\left\|\left(\chi_{Q_{\frac{1}{2}}\left(k^{\prime}\right)} \nabla K\right) *\left(\chi_{Q_{\frac{1}{2}}\left(k^{\prime \prime}\right)} f\right)\right\|_{p} \\
& \leq \sum_{\substack{k^{\prime}, k^{\prime \prime} \in \mathbb{Z}^{n} \\
\max \left|k_{j}^{\prime \prime}+k_{j}^{\prime \prime}-k_{j}\right| \leq 1}}\left\|\chi_{Q_{\frac{1}{2}}\left(k^{\prime}\right)} \nabla K\right\|_{r}\left\|\chi_{Q_{\frac{1}{2}}\left(k^{\prime \prime}\right)} f\right\|_{q} .
\end{aligned}
$$

The set $P(k) \equiv\left\{\left(k^{\prime}, k^{\prime \prime}\right) \in \mathbb{Z}^{n} \times \mathbb{Z}^{n}\left|\max _{1 \leq j \leq n}\right| k_{j}^{\prime}+k_{j}^{\prime \prime}-k_{j} \mid \leq 1\right\}$ for $k=$ $\left(k_{1}, \cdots, k_{n}\right) \in \mathbb{Z}^{n}$ consists of $3^{n}$ elements. Therefore, we obtain

$$
\sum_{\left(k^{\prime}, k^{\prime \prime}\right) \in P(k)}\left\|\chi_{Q_{\frac{1}{2}}\left(k^{\prime}\right)} \nabla K\right\|_{r}\left\|\chi_{Q_{\frac{1}{2}}\left(k^{\prime \prime}\right)} f\right\|_{q} \leq 3^{n} \sup _{k^{\prime \prime} \in \mathbb{Z}^{n}}\|f\|_{L^{q}\left(Q_{\frac{1}{2}}\left(k^{\prime \prime}\right)\right)} \sum_{k^{\prime} \in \mathbb{Z}^{n}}\|\nabla K\|_{L^{r}\left(Q_{\frac{1}{2}}\left(k^{\prime}\right)\right) .} .
$$

Due to the compactness of $Q_{1 / 2}(k)$ for $k \in \mathbb{Z}^{n}$, there exists $y_{k} \in Q_{1 / 2}(k)$ such that $\left|y_{k}\right|=\operatorname{dist}\left(Q_{1 / 2}(k),\{0\}\right)$. If $k=\left(k_{1}, \cdots, k_{n}\right) \in \mathbb{Z}^{n}$ satisfies $\max _{1 \leq j \leq n}\left|k_{j}\right| \geq 2$, it holds that $0 \notin Q_{1 / 2}(k)$. We notice that, for $k \in G \equiv\left\{\ell \in \mathbb{Z}^{n}\left|\max _{1 \leq j \leq n}\right| \ell_{j} \mid \geq 2\right\}$, the function $|\nabla K(x)|$ attains its maximum value at $x=y_{k}$ and we obtain $\|\nabla K\|_{L^{r}\left(Q_{1 / 2}(k)\right)} \leq\left|\nabla K\left(y_{k}\right)\right|$. Thus, we have

$$
\begin{aligned}
\sum_{k \in \mathbb{Z}^{n}}\|\nabla K\|_{L^{r}\left(Q_{\frac{1}{2}}(k)\right)} & =\sum_{k \in G}\|\nabla K\|_{L^{r}\left(Q_{\frac{1}{2}}(k)\right)}+\sum_{k \in G^{c}}\|\nabla K\|_{L^{r}\left(Q_{\frac{1}{2}}(k)\right)} \\
& \leq \sum_{k \in G}\left|\nabla K\left(y_{k}\right)\right|+\sum_{k \in G^{c}}\|\nabla K\|_{L^{r}\left(Q_{\frac{3}{2}}(0)\right)} \\
& =\sum_{k \in G}\left|\nabla K\left(y_{k}\right)\right|+3^{n}\|\nabla K\|_{L^{r}\left(Q_{\frac{3}{2}}(0)\right) .}
\end{aligned}
$$

We define cubes corresponding to $y_{k} \in Q_{1 / 2}(k)$ for $k \in G$. Hence, there exists $\ell \in \mathbb{N}$ and $\left\{k^{j}\right\}_{j=1}^{\ell} \subset \mathbb{Z}^{n}$ such that $Q_{1 / 2}\left(k^{j}\right) \cap\left\{\lambda y_{k}: 0<\lambda<1\right\} \neq \emptyset$ for $j=1, \cdots, \ell$. Here, we denote by $\left\{k^{j}\right\}_{j=1}^{\ell}$ the lattice points such that for $0=\lambda_{0}<\lambda_{1}<\lambda_{2}<$ $\cdots<\lambda_{\ell-1}<\lambda_{\ell}=1, Q_{1 / 2}\left(k^{j}\right)^{\circ} \cap\left\{\lambda y_{k}: 0 \leq \lambda \leq 1\right\}=\left\{\lambda y_{k}: \lambda_{j-1}<\lambda<\lambda_{j}\right\}$. We call such a cube the corresponding cube, and we denote

$$
P_{k} \equiv\left\{k^{\prime} \in \mathbb{Z}^{n} \mid Q_{\frac{1}{2}}\left(k^{\prime}\right) \text { is a corresponding cube such that } Q_{\frac{1}{2}}\left(k^{\prime}\right)=Q_{\frac{1}{2}}(k)\right\}
$$


for $k \in G$. Since $|\nabla K(x)|$ decreases in $|x|$ by Lemma 3.6 and $x \in Q_{1 / 2}\left(k^{j}\right)$ satisfies $|x| \leq\left|y_{k}\right|$, it holds that

$$
\left|\nabla K\left(y_{k}\right)\right|=\int_{Q_{\frac{1}{2}}\left(k^{j}\right)}\left|\nabla K\left(y_{k}\right)\right| \mathrm{d} x \leq \int_{Q_{\frac{1}{2}}\left(k^{j}\right)}|\nabla K(x)| \mathrm{d} x .
$$

By [18, line 5 from above, p.384], for each fixed $k \in G$, the set $P_{k}$ consists of at most $5^{n}$ points. Therefore,

$$
\begin{aligned}
\sum_{k \in G}\left|\nabla K\left(y_{k}\right)\right|+3^{n}\|\nabla K\|_{L^{r}\left(Q_{\frac{3}{2}}(0)\right)} & \leq \sum_{k \in G} \sum_{k^{\prime} \in P_{k}} \int_{Q_{\frac{1}{2}}\left(k^{\prime}\right)}|\nabla K(x)| \mathrm{d} x+3^{n}\|\nabla K\|_{r} \\
& =\sum_{k \in G} \sum_{k^{\prime} \in P_{k}} \int_{Q_{\frac{1}{2}}(k)}|\nabla K(x)| \mathrm{d} x+3^{n}\|\nabla K\|_{r} \\
& \leq 5^{n} \sum_{k \in G} \int_{Q_{\frac{1}{2}}(k)}|\nabla K(x)| \mathrm{d} x+3^{n}\|\nabla K\|_{r} \\
& \leq 5^{n} \int_{\mathbb{R}^{n}}|\nabla K(x)| \mathrm{d} x+3^{n}\|\nabla K\|_{r} .
\end{aligned}
$$

Combining the above computations, we conclude

$$
\|\nabla K * f\|_{L^{p}\left(Q_{\frac{1}{2}}(k)\right)} \leq 3^{n}\left(5^{n}\|\nabla K\|_{1}+3^{n}\|\nabla K\|_{r}\right) \sup _{k^{\prime} \in \mathbb{Z}^{n}}\|f\|_{L^{q}\left(Q_{\frac{1}{2}}\left(k^{\prime}\right)\right)}
$$

and thus

$$
\sup _{k \in \mathbb{Z}^{n}}\|\nabla K * f\|_{L^{p}\left(Q_{\frac{1}{2}}(k)\right)} \leq\left(15^{n}\|\nabla K\|_{1}+9^{n}\|\nabla K\|_{r}\right) \sup _{k^{\prime} \in \mathbb{Z}^{n}}\|f\|_{L^{q}\left(Q_{\frac{1}{2}}\left(k^{\prime}\right)\right)}
$$

where $1+\frac{1}{p}=\frac{1}{q}+\frac{1}{r}$ for $1 \leq r<\frac{n}{n-1}$. Thanks to Lemma 3.7, we obtain

$$
\|\nabla K * f\|_{p, \text { uloc }} \leq\left(90^{n}\|\nabla K\|_{1}+54^{n}\|\nabla K\|_{r}\right)\|f\|_{q, \text { uloc }},
$$

which completes the proof.

Lemma 3.9. For each $p$ satisfying condition (2.4), there exists $k \in(n, \infty]$ and $a$ number $C=C(p, k, n, \nabla K)$ such that

$$
\left\|\nabla e^{\tau \Delta} \cdot(u \nabla K * v)\right\|_{p, \text { uloc }} \leq C \tau^{-\frac{1}{2}}\left(1+\tau^{-\frac{n}{2} \frac{1}{k}}\right)\|u\|_{p, \text { uloc }}\|v\|_{p, \text { uloc }}
$$

for all $u, v \in L_{\mathrm{uloc}}^{p}\left(\mathbb{R}^{n}\right)$ and $\tau>0$.

Proof. Using the heat semigroup estimates from Proposition 3.2 and the Hölder inequality (which also holds in $L_{\text {uloc }}^{p}$-norm), we have

$$
\begin{aligned}
\left\|\nabla e^{\tau \Delta} \cdot(u \nabla K * v)\right\|_{p, \text { uloc }} & \leq C \tau^{-\frac{1}{2}}\left(1+\tau^{-\frac{n}{2} \frac{1}{k}}\right)\|u \nabla K * v\|_{r, \text { uloc }} \\
& \leq C \tau^{-\frac{1}{2}}\left(1+\tau^{-\frac{n}{2} \frac{1}{k}}\right)\|u\|_{p, \text { uloc }}\|\nabla K * v\|_{k, \text { uloc }}
\end{aligned}
$$


where

$$
\frac{1}{r}=\frac{1}{p}+\frac{1}{k} \text { and } \quad r \geq 1
$$

Moreover, applying Proposition 3.8, we obtain

$$
\|\nabla K * v\|_{k, \text { uloc }} \leq C\left(\|\nabla K\|_{1}+\|\nabla K\|_{q_{1}}\right)\|v\|_{p, \text { uloc }}
$$

with

$$
1+\frac{1}{k}=\frac{1}{p}+\frac{1}{q_{1}} \text { and } q_{1} \in\left[1, \frac{n}{n-1}\right) .
$$

Let us show that for every $p$ satisfying condition (2.4) we can always choose $k \in$ $(n, \infty]$ and $r \geq 1$ satisfying the conditions above. Indeed, if $n>1$ then for every $k \in[p, 2 p]$ we have

$$
\frac{1}{q_{1}}=1+\frac{1}{k}-\frac{1}{p} \leq 1 \text { and } \frac{1}{q_{1}}=1+\frac{1}{k}-\frac{1}{p} \geq 1-\frac{1}{2 p}>1-\frac{1}{n} .
$$

Analogously if $n=1$ then the inequalities hold true for every $k \in[p, \infty]$. Next, the condition $r \geq 1$ is equivalent to $k \in[p /(p-1), \infty]$. For $p$ satisfying condition (2.4) and $n>1$, the intersection

$$
[p, 2 p] \cap[p /(p-1), \infty] \cap(n, \infty]
$$

is nonempty, and hence, the choice of $k$ is always possible. Analogously, if $n=1$ we choose arbitrary $k \in[p /(p-1), \infty] \cap(n, \infty]$.

We obtain a solution to integral equation (3.1) from the Banach fixed point theorem formulated in the following way.

Proposition 3.10. Let $X$ be a Banach space and let $Q=Q[\cdot, \cdot]: X \times X \rightarrow X$ be a bounded bilinear form with

$$
\|Q[u, v]\|_{X} \leq C_{1}\|u\|_{X}\|v\|_{X}
$$

for some $C_{1}>0$ independent of $u, v \in X$. Assume that $\delta \in\left(0,1 /\left(4 C_{1}\right)\right)$. If $\left\|y_{0}\right\|_{X} \leq$ $\delta$, then the equation

$$
u=y_{0}+Q[u, u]
$$

has a solution with $\|u\|_{X} \leq 2 \delta$. This solution is unique in the set $\left\{u \in X:\|u\|_{X} \leq 2 \delta\right\}$ and stable in the following sense: if $y_{0}, \tilde{y}_{0} \in X$ satisfy $\left\|y_{0}\right\|_{X} \leq \delta,\left\|\tilde{y}_{0}\right\|_{X} \leq \delta$ then for the corresponding solutions $u, \tilde{u} \in X$ we have

$$
\|u-\widetilde{u}\|_{X} \leq C_{2}\left\|y_{0}-\tilde{y}_{0}\right\|_{X}
$$

where $C_{2}>0$ is independent of $u$ and $\tilde{u}$. 
Proof of Theorem 2.1. For $T>0$, we introduce $X_{T} \equiv L^{\infty}\left((0, T) ; L_{\mathrm{uloc}}^{p}\left(\mathbb{R}^{n}\right)\right)$ which

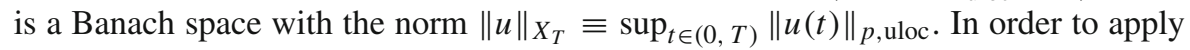
Proposition 3.10, it suffices to estimate the bilinear form

$$
Q[u, v](t)=-\int_{0}^{t} \nabla e^{(t-s) \Delta} \cdot(u(s) \nabla K * v(s)) \mathrm{d} s .
$$

By Lemma 3.9, for some $k>n$, we obtain

$$
\begin{aligned}
\|Q[u, v](t)\|_{p, \mathrm{uloc}} & \leq \int_{0}^{t}\left\|\nabla e^{(t-s) \Delta} \cdot(u(s) \nabla K * v(s))\right\|_{p, \text { uloc }} \\
& \leq C \int_{0}^{t}(t-s)^{-\frac{1}{2}}\left(1+(t-s)^{-\frac{n}{2} \frac{1}{k}}\right)\|u(s)\|_{p, \text { uloc }}\|v(s)\|_{p, \text { uloc }} \mathrm{d} s \\
& \leq C\|u\|_{X_{T}}\|v\|_{X_{T}} \int_{0}^{t}(t-s)^{-\frac{1}{2}}\left(1+(t-s)^{-\frac{n}{2} \frac{1}{k}}\right) \mathrm{d} s \\
& \leq C\left(t^{\frac{1}{2}}+t^{\frac{1}{2}-\frac{n}{2 k}}\right)\|u\|_{X_{T}}\|v\|_{X_{T}}
\end{aligned}
$$

Therefore, we have

$$
\|Q[u, v]\|_{X_{T}} \leq C\left(T^{\frac{1}{2}}+T^{\frac{1}{2}-\frac{n}{2 k}}\right)\|u\|_{X_{T}}\|v\|_{X_{T}} .
$$

Since inequality (3.4) in Proposition 3.2 provides $\left\|e^{t \Delta} u_{0}\right\|_{X_{T}} \leq\left\|u_{0}\right\|_{p \text {, uloc }}$, we obtain a solution to the integral equation via Proposition 3.10 for sufficiently small $T>0$. In order to show that $u \in C\left((0, T) ; L_{\mathrm{uloc}}^{p}\left(\mathbb{R}^{n}\right)\right)$ it suffices to follow the arguments from [18, p. 388]. This solution is unique by a usual reasoning.

To show that a solution is nonnegative in the case of nonnegative initial datum, we pass through an approximation process with a sequence of smooth solutions $\left\{u^{\varepsilon}\right\}_{\varepsilon>0}$ corresponding to the smooth, uniformly bounded nonnegative initial conditions $u_{0}^{\varepsilon}(x)=\left(e^{\varepsilon \Delta} u_{0}\right)(x)$. Here, $u^{\varepsilon} \geq 0$ by the classical maximum principle, see $e . g$. [9, Theorem 9, p.43]. To complete this reasoning, we show that $\left\|u^{\varepsilon}(t)-u(t)\right\|_{p, \text { uloc }} \rightarrow$ 0 as $\varepsilon \rightarrow 0$ for each $t \in(0, T)$. Indeed, from inequality (3.4) in Proposition 3.2, it holds that $\left\|u_{0}^{\varepsilon}\right\|_{p \text {,uloc }} \leq\left\|u_{0}\right\|_{p \text {, uloc }}$ for all $\varepsilon>0$. Hence, by the above construction of a local-in-time solutions via Proposition 3.10, there exists a constant $M>0$ such that $\|u\|_{X_{T}} \leq M$ and $\left\|u^{\varepsilon}\right\|_{X_{T}} \leq M$. Now, computing the norm $\left\|u^{\varepsilon}(t)-u(t)\right\|_{p, \text { uloc }}$ and using the integral representation (3.1) of these functions as well as a second inequality in estimate (3.6), we obtain

$$
\begin{aligned}
& \left\|u^{\varepsilon}(t)-u(t)\right\|_{p, \text { uloc }} \\
& \quad \leq\left\|e^{t \Delta} u_{0}^{\varepsilon}-e^{t \Delta} u_{0}\right\|_{p, \text { uloc }} \\
& \quad+C\left(\left\|u^{\varepsilon}\right\|_{X_{T}}+\|u\|_{X_{T}}\right) \int_{0}^{t}(t-s)^{-\frac{1}{2}}\left(1+(t-s)^{-\frac{n}{2} \frac{1}{k}}\right)\left\|u^{\varepsilon}(s)-u(s)\right\|_{p, \text { uloc }} \mathrm{d} s .
\end{aligned}
$$


Applying the Volterra-type inequality from [25, Ch. 9] we conclude that $\left\|u^{\varepsilon}(t)-u(t)\right\|_{p, \text { uloc }} \leq\left\|e^{t \Delta} u_{0}^{\varepsilon}-e^{t \Delta} u_{0}\right\|_{p, \text { uloc }}+C \int_{0}^{t} \kappa(t-s)\left\|e^{s \Delta} u_{0}^{\varepsilon}-e^{s \Delta} u_{0}\right\|_{p, \text { uloc }} \mathrm{d} s$, where $\kappa=\kappa(\tau)$ is a suitable integrable kernel on $[0, t]$. Finally, by Remark 3.4 and the semigroup properties, we have

$$
\left\|e^{t \Delta} u_{0}^{\varepsilon}-e^{t \Delta} u_{0}\right\|_{p, \text { uloc }}=\left\|e^{\varepsilon \Delta} e^{t \Delta} u_{0}-e^{t \Delta} u_{0}\right\|_{p, \text { uloc }} \rightarrow 0 \text { as } \varepsilon \rightarrow 0 .
$$

\section{Linearized problem}

\subsection{Preliminary properties}

The linearization procedure described below in Sect. 5 leads to the following linear problem

$$
\left\{\begin{array}{l}
v_{t}-\Delta v+A \Delta K * v=0, \quad t>0, \quad x \in \mathbb{R}^{n} \\
v(0, x)=v_{0}(x)
\end{array}\right.
$$

where $A \in \mathbb{R}$ is an arbitrary constant and the operator $\Delta-A \Delta K *$ can be expressed by the Fourier transform as follows

$$
(\Delta \varphi-A \Delta K * \varphi) \widehat{\Im}(\xi)=\left(-|\xi|^{2}+A \frac{|\xi|^{2}}{1+|\xi|^{2}}\right) \widehat{\varphi}(\xi), \quad \xi \in \mathbb{R}^{n} .
$$

We begin by presenting preliminary properties of this operator.

Lemma 4.1. There exists a constant $L>0$ such that for each $p \in[1, \infty]$,

$$
\|-\Delta K * v\|_{p} \leq L\|v\|_{p} \quad \text { for all } \quad v \in L^{p}\left(\mathbb{R}^{n}\right) .
$$

This lemma is an immediate consequence of the fact that the operator $-\Delta K *$ can be represented by a convolution with a finite measure on $\mathbb{R}^{n}$. We skip the proof of this classical result from the harmonic analysis, see e.g. [21, Lemma 2.(i), p.133].

Lemma 4.2. For each $A \in \mathbb{R}$, a closure in $L^{p}\left(\mathbb{R}^{n}\right)$ of the operator $\Delta-A \Delta K *$ generates an analytic semigroup $\left\{S_{A}(t)\right\}_{t \geq 0}$ on $L^{p}\left(\mathbb{R}^{n}\right)$ for every $p \in[1, \infty)$. This semigroup is defined by the Fourier transform by the formula

$$
\widehat{S_{A}(t) v_{0}}(\xi)=\widehat{\mu}_{A}(t, \xi) \widehat{v_{0}}(\xi)
$$

where

$$
\widehat{\mu}_{A}(t, \xi)=e^{-t\left(|\xi|^{2}-A \frac{|\xi|^{2}}{1+|\xi|^{2}}\right)} .
$$


Proof. It is well-known that Laplacian generates an analytic semigroup of linear operators on $L^{p}\left(\mathbb{R}^{n}\right)$ for every $p \in[1, \infty)$. A bounded perturbation of such an operator maintains the same property, see e.g. [7, Chapter III, Theorem 2.10]. The Fourier representation of this semigroup can be obtained by routine calculations.

Lemma 4.3. Assume that $A \in \mathbb{R}$ and choose the constant $L$ from inequality (4.2). Then for each $1 \leq q \leq p \leq \infty$, there exists a constant $C=C(p, q, n)>0$ such that

$$
\left\|S_{A}(t) v_{0}\right\|_{p} \leq C t^{-\frac{n}{2}\left(\frac{1}{q}-\frac{1}{p}\right)} e^{|A| L t}\left\|v_{0}\right\|_{q}
$$

and

$$
\left\|\nabla S_{A}(t) v_{0}\right\|_{p} \leq C t^{-\frac{n}{2}\left(\frac{1}{q}-\frac{1}{p}\right)-\frac{1}{2}} e^{|A| L t}\left\|v_{0}\right\|_{q}
$$

for all $t>0$ and $v_{0} \in L^{q}\left(\mathbb{R}^{n}\right)$.

Proof. Here, we use the notation $S_{A}(t) v_{0}=e^{t \Delta}\left(e^{-A \Delta K *} v_{0}\right)$. Using the $L^{p}-L^{q}$ estimates of the heat semigroup (see Remark 3.3) and Lemma 4.1, we obtain

$$
\left\|e^{t \Delta}\left(e^{-t A \Delta K *} v_{0}\right)\right\|_{p} \leq C t^{-\frac{n}{2}\left(\frac{1}{q}-\frac{1}{p}\right)}\left\|e^{-t A \Delta K *} v_{0}\right\|_{q} \leq C t^{-\frac{n}{2}\left(\frac{1}{q}-\frac{1}{p}\right)} e^{|A| L t}\left\|v_{0}\right\|_{q} .
$$

The proof for the second inequality is analogous.

\subsection{Decay estimates when $A<1$}

The following theorem improves estimates from Lemma 4.3 in the case of $A \in$ $[0,1)$, and it plays a crucial role in the proof of stability of constant solutions to problem (2.2).

Theorem 4.4. Assume that $A \in[0,1)$. For all exponents satisfying $1 \leq q \leq p \leq \infty$, there exist constants $C=C(p, q, n, A)>0$ such that

$$
\left\|S_{A}(t) v_{0}\right\|_{p} \leq C t^{-\frac{n}{2}\left(\frac{1}{q}-\frac{1}{p}\right)}\left\|v_{0}\right\|_{q}
$$

and

$$
\left\|\nabla S_{A}(t) v_{0}\right\|_{p} \leq C t^{-\frac{n}{2}\left(\frac{1}{q}-\frac{1}{p}\right)-\frac{1}{2}}\left\|v_{0}\right\|_{q}
$$

for all $t>0$ and $v_{0} \in L^{q}\left(\mathbb{R}^{n}\right)$.

The proof of this theorem is based on the following lemmas.

Lemma 4.5. Let $\widehat{D^{N} v}(\xi) \equiv|\xi|^{N} \widehat{v}(\xi)$ for all $N \in \mathbb{R}$. For all $v \in \mathscr{S}\left(\mathbb{R}^{n}\right)$ (the Schwartz class of smooth rapidly decreasing functions) and for every $N>\frac{n}{2}$, the following inequality holds

$$
\|v\|_{1} \leq C\|\widehat{v}\|_{2}^{1-\frac{n}{2 N}}\left\|D^{N} \widehat{v}\right\|_{2}^{\frac{n}{2 N}},
$$

with a constant $C=C(n, N)>0$. 
Proof. For $R=\left(\frac{2 N-n}{n} \frac{\left\|D^{N} \widehat{v}\right\|_{2}}{\|\widehat{v}\|_{2}}\right)^{1 / N}$, we obtain

$$
\begin{aligned}
\|v\|_{1}= & \int_{|x| \leq R}|v(x)| \mathrm{d} x+\int_{|x|>R}|v(x)| \mathrm{d} x \\
\leq & \left(\int_{|x| \leq R} \mathrm{~d} x\right)^{\frac{1}{2}}\left(\int_{|x| \leq R}|v(x)|^{2} \mathrm{~d} x\right)^{\frac{1}{2}} \\
& +\left(\int_{|x|>R}|x|^{-2 N} \mathrm{~d} x\right)^{\frac{1}{2}}\left(\int_{|x|>R}|x|^{2 N}|v(x)|^{2} \mathrm{~d} x\right)^{\frac{1}{2}} \\
\leq & \left|\mathbb{S}^{n-1}\right|^{\frac{1}{2}}\left(R^{\frac{n}{2}}\|v\|_{2}+R^{\frac{n}{2}-N}\left\|D^{N} \widehat{v}\right\|_{2}\right) \\
\leq & C\|\widehat{v}\|_{2}^{1-\frac{n}{2 N}}\left\|D^{N} \widehat{v}\right\|_{2}^{\frac{n}{2 N}} .
\end{aligned}
$$

Lemma 4.6. Assume that $A \in[0,1)$. For the function $\widehat{\mu}_{A}$ defined by formula (4.3) and for every multi-index $\alpha$ with $|\alpha|=N$, there exists a constant $C=C(n, A, N)>0$ such that

$$
\left\|\partial_{\xi}^{\alpha} \widehat{\mu}_{A}(t)\right\|_{2}^{2} \leq C t^{N-\frac{n}{2}}, \text { for all } t \geq 1 \text {. }
$$

Proof. For $N=0$, by the inequality $|\xi|^{2} /\left(1+|\xi|^{2}\right) \leq|\xi|^{2}\left(\xi \in \mathbb{R}^{n}\right)$, we obtain

$$
\left\|\widehat{\mu}_{A}(t)\right\|_{2}^{2}=\int_{\mathbb{R}^{n}} e^{-2 t|\xi|^{2}+2 A t \frac{|\xi|^{2}}{1+|\xi|^{2}}} \mathrm{~d} \xi \leq \int_{\mathbb{R}^{n}} e^{-2 t(1-A)|\xi|^{2}} \mathrm{~d} \xi=C t^{-\frac{n}{2}} .
$$

For $N \geq 1$, we introduce the $C^{\infty}$-function $h(\xi) \equiv|\xi|^{2}-A|\xi|^{2} /\left(1+|\xi|^{2}\right)$, which satisfies estimates $\left|\partial_{\xi_{j}} h(\xi)\right| \leq C|\xi|$ and $\left|\partial_{\xi}^{\beta} h(\xi)\right| \leq C$ for every $j, 1 \leq j \leq n$ and multi-index $\beta$ with $|\beta| \geq 2$. We use the multivariate Faà di Bruno's formula (see e.g. [11])

$$
\partial_{\xi}^{\alpha} e^{-t h(\xi)}=e^{-t h(\xi)} \sum_{k=1}^{N}(-t)^{k} H_{k}(\xi),
$$

where $H_{k}(\xi)$ is a sum of products of $k$ partial derivatives of the function $h(\xi)$ such that $\left|H_{k}(\xi)\right| \leq C\left(1+|\xi|^{k}\right)$. We prove the following inequality by induction in $N \in \mathbb{N}$,

$$
\left|\partial_{\xi}^{\alpha} e^{-t h(\xi)}\right| \leq C e^{-t h(\xi)} \sum_{k-\frac{\ell}{2} \leq \frac{N}{2}} t^{k}\left(1+|\xi|^{\ell}\right) .
$$

For $N=1$, the inequality is obvious. We show the induction step for $N+1$,

$$
\begin{aligned}
\left|\partial_{\xi_{j}} \partial_{\xi}^{\alpha} e^{-t h(\xi)}\right| & \leq t\left|\partial_{\xi_{j}} h(\xi)\right|\left|\partial_{\xi}^{\alpha} e^{-t h(\xi)}\right|+\left|e^{-t h(\xi)} \sum_{k=1}^{N}(-t)^{k} \partial_{\xi_{j}} H_{k}(\xi)\right| \\
& \leq C t|\xi| e^{-t h(\xi)} \sum_{k-\frac{\ell}{2} \leq \frac{N}{2}} t^{k}\left(1+|\xi|^{\ell}\right)+C e^{-t h(\xi)} \sum_{k-\frac{\ell}{2} \leq \frac{N}{2}} t^{k}\left(1+|\xi|^{\ell}\right) \\
& \leq C e^{-t h(\xi)} \sum_{k-\frac{\ell}{2} \leq \frac{N+1}{2}} t^{k}\left(1+|\xi|^{\ell}\right)
\end{aligned}
$$


which holds true because $\left|\partial_{\xi_{j}} H_{k}(\xi)\right| \leq C\left(1+|\xi|^{\ell}\right)$ by the properties of the function $h(\xi)$ and $k+1-(\ell+1) / 2 \leq(N+1) / 2$.

Now we group coefficients $t^{k}$ and $|\xi|^{\ell}$ in the following way, $t^{k}|\xi|^{\ell}=t^{k-\ell / 2}|\sqrt{t} \xi|^{\ell}$ thus, by the assumption $t \geq 1$ and induction, we have $t^{k-\ell / 2} \leq t^{N / 2}$. We obtain an estimate

$$
\left|\partial_{\xi}^{\alpha} \widehat{\mu}_{A}(t)\right| \leq C t^{\frac{N}{2}} P(|\sqrt{t} \xi|) e^{-t|\xi|^{2}+A t \frac{|\xi|^{2}}{1+|\xi|^{2}}},
$$

where $P(s)$ is a polynomial of degree $N$. By the same inequality as in the case $N=0$ and properties of the exponential function,

$$
\left|\partial_{\xi}^{\alpha} \widehat{\mu}_{A}(t)\right| \leq t^{\frac{N}{2}} P(|\sqrt{t} \xi|) e^{-t(1-A)|\xi|^{2}} \leq C t^{\frac{N}{2}} e^{-\delta t|\xi|^{2}},
$$

for some $\delta \in(0,1-A)$. Calculating the $L^{2}$-norm of both sides of inequality (4.6), we obtain the result.

Lemma 4.7. Assume that $A \in[0,1)$. For every $p \in[1, \infty]$, there exists a constant $C>0$ such that

$$
\left\|S_{A}(t) v_{0}\right\|_{p} \leq C\left\|v_{0}\right\|_{p},
$$

for all $t>0$ and all $v_{0} \in L^{p}\left(\mathbb{R}^{n}\right)$.

Proof. For $t \in[0,1]$, this is an immediate consequence of Lemma 4.3. For $t \geq 1$, the function $\mu_{A}$ is from the Schwartz class in variable $\xi$. Thus, by the Young inequality,

$$
\left\|S_{A}(t) v_{0}\right\|_{p}=\left\|\mu_{A}(t) * v_{0}\right\|_{p} \leq\left\|\mu_{A}(t)\right\|_{1}\left\|v_{0}\right\|_{p} .
$$

In order to estimate $\left\|\mu_{A}(t)\right\|_{1}$, we recall a well-known fact that both quantities $\left\|D^{N} v\right\|_{2}$ and $\sum_{|\alpha|=N}\left\|\partial_{x}^{\alpha} v\right\|_{2}$ are comparable for each $N \in \mathbb{N}$. Combining Lemmas 4.5 and 4.6, for $N>n / 2$ and $N \in \mathbb{N}$, we obtain

$$
\left\|\mu_{A}(t)\right\|_{1} \leq C\left\|\widehat{\mu}_{A}(t)\right\|_{2}^{1-\frac{n}{2 N}}\left\|D^{N} \widehat{\mu}_{A}(t)\right\|_{2}^{\frac{n}{2 N}} \leq C\left(C_{1} t^{-\frac{n}{4}}\right)^{1-\frac{n}{2 N}}\left(C_{2} t^{\frac{N}{2}-\frac{n}{4}}\right)^{\frac{n}{2 N}}=C
$$

for all $t \geq 1$.

Proof of Theorem 4.4. We begin with inequality (4.4). Let us choose $\varepsilon \in(A, 1)$. By the standard heat semigroup estimates (see Remark 3.3),

$$
\left\|S_{A}(t) v_{0}\right\|_{p}=\left\|e^{(1-\varepsilon) t \Delta}\left(e^{\varepsilon t \Delta-t A \Delta K *} v_{0}\right)\right\|_{p} \leq C_{1} t^{-\frac{n}{2}\left(\frac{1}{q}-\frac{1}{p}\right)}\left\|e^{\varepsilon t \Delta-t A \Delta K *} v_{0}\right\|_{q} .
$$

Now we substitute $\tilde{t}=\varepsilon t$ to obtain $t A=\tilde{t}(A / \varepsilon)=\tilde{t} \tilde{A}$ and $0 \leq \tilde{A}<1$. Thus, by Lemma 4.7,

$$
\left\|S_{A}(t) v_{0}\right\|_{p} \leq C_{1} t^{-\frac{n}{2}\left(\frac{1}{q}-\frac{1}{p}\right)}\left\|e^{\tilde{t} \Delta-\tilde{t} \tilde{A} \Delta K *} v_{0}\right\|_{q} \leq C t^{-\frac{n}{2}\left(\frac{1}{q}-\frac{1}{p}\right)}\left\|v_{0}\right\|_{q} .
$$

We prove inequality (4.5) analogously using the formula

$$
\nabla S_{A}(t) v_{0}=\nabla e^{(1-\varepsilon) t \Delta}\left(e^{\varepsilon t \Delta-t A \Delta K *} v_{0}\right)
$$


Remark 4.8. The $L^{q}-L^{p}$ estimates (4.4)-(4.5) of the semigroup $\left\{S_{A}(t)\right\}_{t>0}$ hold true for $A<0$ as well. They can be proved by the same reasoning as above using the obvious inequality

$$
e^{-t\left(|\xi|^{2}-A \frac{|\xi|^{2}}{1+|\xi|^{2}}\right)} \leq e^{-t|\xi|^{2}} \text { for each } A<0 .
$$

Remark 4.9. For the completeness of this work, we notice that constant solution $A=1$ is linearly stable in $L^{2}\left(\mathbb{R}^{n}\right)$. Indeed, since $e^{-t\left(|\xi|^{2}-|\xi|^{2} /\left(1+|\xi|^{2}\right)\right)} \leq 1$ for all $\xi \in \mathbb{R}^{n}$ and $t \geq 0$, by the Plancherel formula, we obtain $\left\|S_{1}(t) v_{0}\right\|_{2} \leq\left\|v_{0}\right\|_{2}$ for all $v_{0} \in L^{2}\left(\mathbb{R}^{n}\right)$. We skip a discussion of a stability of this constant solution for $p \neq 2$.

\subsection{Exponential growth when $A>1$}

Next, we study an instability of solutions to linear problem (4.1). In Lemma 4.10, we show that the spectrum of the operator $\Delta-A \Delta K *$ contains positive elements for $A>1$. Lemma 4.11 describes estimates of the semigroup involving positive spectral bound of this operator. Lemma 4.12 states that estimates of this semigroup are optimal.

Lemma 4.10. Let $p \in(1, \infty)$. The closure in $L^{p}\left(\mathbb{R}^{n}\right)$ of the operator $\Delta-A \Delta K *$ has a real continuous spectrum $(-\infty, a]$, where $a=0$ if $A \leq 1$ and $a=(\sqrt{A}-1)^{2}$ if $A>1$.

Proof. The symbol $h(\xi)=|\xi|^{2}-A|\xi|^{2} /\left(1+|\xi|^{2}\right)$ of the operator satisfies the estimates

$$
\left|\partial_{\xi}^{\alpha} h(\xi)\right| \leq C_{\alpha}(1+|\xi|)^{2-|\alpha|} \text { for all } \xi \in \mathbb{R}^{n}
$$

and each multi-index $\alpha=\left(\alpha_{1}, \ldots, \alpha_{n}\right)$. Moreover, $h(\xi)^{-1}=O\left(|\xi|^{-2}\right)$ as $|\xi| \rightarrow \infty$. Now, it suffices to apply the result from [24, Theorem 2.1].

Lemma 4.11. Assume $A>1, a=(\sqrt{A}-1)^{2}$ and $1<q \leq p<\infty$. For every $\varepsilon>0$ there exists a constant $C=C(\varepsilon, p, q, A)>0$ such that

$$
\left\|S_{A}(t) v_{0}\right\|_{p} \leq C t^{-\frac{n}{2}\left(\frac{1}{q}-\frac{1}{p}\right)} e^{(a+\varepsilon) t}\left\|v_{0}\right\|_{q}
$$

and

$$
\left\|\nabla S_{A}(t) v_{0}\right\|_{p} \leq C t^{-\frac{n}{2}\left(\frac{1}{q}-\frac{1}{p}\right)-\frac{1}{2}} e^{(a+\varepsilon) t}\left\|v_{0}\right\|_{q}
$$

for all $v_{0} \in L^{q}\left(\mathbb{R}^{n}\right)$ and $t>0$.

Proof. Estimate (4.7) for $p=q$ is a direct consequence of Lemma 4.10 combined with estimates of strongly continuous semigroups, see e.g. [7, Ch.IV, Corollary 3.12]. In order to prove inequality (4.8) for $p=q$, notice that for every $\delta \in(0,1)$ we have

$$
\nabla S_{A}(t) v_{0}=\nabla\left(e^{\delta t \Delta}\left(e^{t((1-\delta) \Delta-A \Delta K *)}\right)\right) v_{0}=\nabla e^{\delta t \Delta}\left(e^{t(1-\delta)\left(\Delta-\frac{A}{1-\delta} \Delta K *\right)} v_{0}\right)
$$


Applying the estimates of the heat semigroup from Remark 3.3 and inequality (4.7) with $\varepsilon / 2$ to the operator $S_{\frac{A}{1-\delta}}(t)$ yields

$\left\|\nabla S_{A}(t) v_{0}\right\|_{p} \leq C(\varepsilon / 2)(\delta t)^{-1 / 2} e^{t(1-\delta)\left(a_{\delta}+\varepsilon / 2\right)}\left\|v_{0}\right\|_{p} \quad$ with $\quad a_{\delta}=\left(\sqrt{\frac{A}{1-\delta}}-1\right)^{2}$.

Choosing $\delta>0$ sufficiently small to have $a_{\delta} \leq a+\varepsilon / 2$.

For $q<p$, we proceed analogously using $L^{q}-L^{p}$ estimates of the heat semigroup from Remark 3.3.

Lemma 4.12. Assume $A>1, a=(\sqrt{A}-1)^{2}$ and $p \in(1, \infty)$. For every $\gamma \in(0,1]$ and every $T>0$, there exists $v_{0} \in L^{p}\left(\mathbb{R}^{n}\right)$ such that for each $t \in[0, T]$

$$
\left\|S_{A}(t) v_{0}-e^{a t} v_{0}\right\|_{p} \leq \gamma\left\|v_{0}\right\|_{p} \quad \text { and } \quad\left\|S_{A}(t) v_{0}\right\|_{p} \leq 2 e^{a t}\left\|v_{0}\right\|_{p} .
$$

Proof. It follows from Lemma 4.10 that $a=(\sqrt{A}-1)^{2}$ lies on the boundary of the spectrum of the operator $\Delta-A \Delta K *$ and such elements belong to the approximate point spectrum (see e.g. [20, Lemma 1]). Thus, for each $\varepsilon>0$ there exists $v_{\varepsilon} \in L^{p}\left(\mathbb{R}^{n}\right)$ (in fact, $v_{\varepsilon}$ belongs to the domain of the closure in $L^{p}\left(\mathbb{R}^{n}\right)$ of the operator $\Delta-A \Delta K *$ ) such that

$$
\left\|\Delta v_{\varepsilon}-A \Delta K * v_{\varepsilon}-a v_{\varepsilon}\right\|_{p} \leq \varepsilon\left\|v_{\varepsilon}\right\|_{p} .
$$

Hence, by usual calculations for semigroups of linear operators (see e.g. [7, Ch.II, Sec. 3]) and by Lemma 4.3, we obtain

$$
\begin{aligned}
\left\|S_{A}(t) v_{\varepsilon}-e^{a t} v_{\varepsilon}\right\|_{p} & =\left\|\int_{0}^{1} \frac{\mathrm{d}}{\mathrm{d} s} e^{t s(\Delta-A \Delta K *)} e^{t(1-s) a} v_{\varepsilon} \mathrm{d} s\right\|_{p} \\
& \leq \int_{0}^{1}\left\|e^{t s(\Delta-A \Delta K *)} e^{t(1-s) a} t\left(\Delta v_{\varepsilon}-A \Delta K * v_{\varepsilon}-a v_{\varepsilon}\right)\right\|_{p} \mathrm{~d} s \\
& \leq C t\left\|\Delta v_{\varepsilon}-A \Delta K * v_{\varepsilon}-a v_{\varepsilon}\right\|_{p} \int_{0}^{1} e^{|A| L t s} e^{t(1-s) a} \mathrm{~d} s,
\end{aligned}
$$

for some constant $C>0$ from inequality (4.3) with $p=q$. Therefore, inequality (4.10) with $\varepsilon=\frac{\gamma}{C T e^{(|A| L+a) T}}$ provides the estimate

$$
\left\|S_{A}(t) v_{0}-e^{a t} v_{0}\right\|_{p} \leq \frac{t e^{(|A| L+a) t}}{T e^{(|A| L+a) T}} \gamma\left\|v_{0}\right\|_{p} \leq \gamma\left\|v_{0}\right\|_{p} .
$$

Since $a>0$, the second inequality in (4.9) can be obtained immediately from the first one by choosing $\gamma=1$.

\section{Perturbations of constant solutions}

We study a solution $u=u(t, x)$ of problem (2.2), which is a perturbation of the constant stationary solution $A \in \mathbb{R}$. Thus, the function $v(t, x)=u(t, x)-A$ satisfies

$$
\begin{cases}v_{t}-\Delta v+A \Delta K * v+\nabla \cdot(v \nabla K * v)=0, & t>0, \quad x \in \mathbb{R}^{n}, \\ v(0, x)=v_{0}(x), & x \in \mathbb{R}^{n} .\end{cases}
$$


Here, in fact, we consider a mild solution to this problem satisfying the integral equation

$$
v(t)=S_{A}(t) v_{0}-\int_{0}^{t} \nabla S_{A}(t-\tau) \cdot(v(\tau) \nabla K * v(\tau)) \mathrm{d} \tau,
$$

where the semigroup $\left\{S_{A}(t)\right\}_{t \geq 0}$ has been studied in Sect. 4. In particular, this semigroup commutes with $\nabla$ by the same reason as stated in Remark 3.1.

\subsection{Local-in-time solutions}

We begin by a result on local-in-time solutions.

Proposition 5.1. For each $p$ satisfying condition (2.5) and every $v_{0} \in L^{p}\left(\mathbb{R}^{n}\right)$, there exists $T>0$ and a unique local-in-time mild solution to problem (5.1) in $C\left([0, T) ; L^{p}\left(\mathbb{R}^{n}\right)\right)$.

For the proof of this proposition, one should follow the reasoning in the proof of Theorem 2.1. In particular, a solution is obtained via Proposition 3.10 where the required estimate of the bilinear form

$$
\widetilde{Q}[v, w](t)=-\int_{0}^{t} \nabla S_{A}(t-s) \cdot(v(s) \nabla K * w(s))(s) \mathrm{d} s
$$

is a direct consequence of the following lemma.

Lemma 5.2. For each $p$ satisfying condition (2.5), there exist $k \in(n, \infty]$ and positive numbers $C_{1}=C_{1}(p, k, n, \nabla K)$ and $C_{2}=C_{2}(p, k, n, \nabla K)$ such that

$$
\left\|\nabla S_{A}(\tau) \cdot(v \nabla K * w)\right\|_{p} \leq C_{2} \tau^{-\frac{n}{2} \frac{1}{k}-\frac{1}{2}} e^{C_{1} \tau}\|v\|_{p}\|w\|_{p} \text { for all } v, w \in L^{p}\left(\mathbb{R}^{n}\right) .
$$

If $A<1$ then $C_{1}=0$ and if $A \geq 1$ then $C_{1}$ can be an arbitrary constant satisfying $C_{1}>a=(\sqrt{A}-1)^{2}$.

We skip the proof of this lemma, because it is the same as the proof of Lemma 3.9. In particular, it is based on the semigroup estimates from Lemma 4.11.

Remark 5.3. Notice that for every $A \in \mathbb{R}$ and $v_{0} \in L^{p}\left(\mathbb{R}^{n}\right)$ we have $A+v_{0} \in$ $\mathscr{L}_{\text {uloc }}^{p}\left(\mathbb{R}^{n}\right)$ which is an immediate consequence of Remark 3.3 combined with the relation

$$
\left\|e^{t \Delta}\left(A+v_{0}\right)-\left(A+v_{0}\right)\right\|_{p, \mathrm{uloc}} \leq\left\|e^{t \Delta} v_{0}-v_{0}\right\|_{p} \rightarrow 0 \text { as } t \rightarrow 0
$$

5.2. Global-in-time solutions for $A \in[0,1)$

The proof of Theorem 2.3 requires the following extension of Lemma 5.2.

Lemma 5.4. Assume that $A \in[0,1)$. For 
- each p satisfying condition (2.5),

- each $q>n$ if $n \geq 2$ and $q \geq 1$ if $n=1$ satisfying $q \in[p, 2 p]$,

- each $r \geq 1$ such that $r \in\left[\frac{q}{2}, p\right]$,

there exists a constant $C>0$ such that

$$
\left\|\nabla S_{A}(\tau) \cdot(v \nabla K * w)\right\|_{p} \leq C \tau^{-\frac{n}{2}\left(\frac{1}{r}-\frac{1}{p}\right)-\frac{1}{2}}\|v\|_{q}\|w\|_{q},
$$

for all $v, w \in L^{q}\left(\mathbb{R}^{n}\right)$ and $\tau>0$.

Proof. First, we use Theorem 4.4 and the Hölder inequality to estimate

$\left\|\nabla S_{A}(\tau) \cdot(v \nabla K * w)\right\|_{p} \leq C \tau^{-\frac{n}{2}\left(\frac{1}{r}-\frac{1}{p}\right)-\frac{1}{2}}\|v \nabla K * w\|_{r} \leq C \tau^{-\frac{n}{2}\left(\frac{1}{r}-\frac{1}{p}\right)-\frac{1}{2}}\|v\|_{q}\|\nabla K * w\|_{k}$

with $1 \leq r \leq p$ satisfying $\frac{1}{r}=\frac{1}{q}+\frac{1}{k}$.

Next, we apply the Young inequality

$$
\|\nabla K * w\|_{k} \leq C\|\nabla K\|_{q_{1}}\|w\|_{q}
$$

with $\frac{1}{q_{1}}+\frac{1}{q}=1+\frac{1}{k}$. Let us show that $q_{1} \in\left[1, \frac{n}{n-1}\right)$ in order to have $\nabla K \in L^{q_{1}}\left(\mathbb{R}^{n}\right)$. Indeed, by the assumption on $p, q, r$ we have

$$
1-\frac{1}{q} \leq \frac{1}{q_{1}}=1+\frac{1}{r}-\frac{2}{q} \leq 1,
$$

where, for $n \geq 2$, we use also the inequality $1-\frac{1}{q}>1-\frac{1}{n}$.

Proof of Theorem 2.3. It is sufficient to construct a global-in-time solution to problem (5.1) formulated in the mild form (5.2), because $u=A+v$ by the uniqueness of solutions from Proposition 5.1. The solution is obtained via Proposition 3.10 applied to equation (5.2) in the Banach space

$$
\mathscr{X}=C\left([0, \infty) ; L^{p}\left(\mathbb{R}^{n}\right)\right) \cap\left\{v \in C\left((0, \infty) ; L^{q}\left(\mathbb{R}^{n}\right)\right): \sup _{t>0} t^{\frac{n}{2}\left(\frac{1}{p}-\frac{1}{q}\right)}\|v(t)\|_{q}<\infty\right\}
$$

with the norm $\|v\|_{\mathscr{X}} \equiv \sup _{t>0}\|v(t)\|_{p}+\sup _{t>0} t^{\frac{n}{2}\left(\frac{1}{p}-\frac{1}{q}\right)}\|v(t)\|_{q}$.

First, for every $v_{0} \in L^{p}\left(\mathbb{R}^{n}\right)$, it follows from Theorem 4.4 that

$$
\left\|S_{A}(t) v_{0}\right\| \mathscr{X} \leq C\left\|v_{0}\right\|_{p}
$$

for all $t>0$ and for some constant $C=C(p, q, r, n)>0$.

Next, we estimate the bilinear form $\widetilde{Q}[u, v]$ given by formula (5.3) for all $v, w \in \mathscr{X}$. Here, for $p$ satisfying condition (2.4) with $p \leq n$ and for $q \in(n, 2 p]$, we choose one more exponent $r \in\left[\frac{q}{2}, p\right]$ and $r \geq 1$ such that

$$
\frac{2}{q}+\frac{1}{n}-\frac{1}{p} \leq \frac{1}{r} \leq \frac{2}{q}
$$


By Lemma 5.2, there exists $k>n$ such that

$$
\|\widetilde{Q}[v, w](t)\|_{p} \leq C \int_{0}^{t}(t-s)^{-\frac{n}{2} \frac{1}{k}-\frac{1}{2}}\|v(s)\|_{p}\|w(s)\|_{p} \mathrm{~d} s \leq C t^{-\frac{n}{2 k}+\frac{1}{2}}\|v\|_{\mathscr{X}}\|w\|_{\mathscr{X}}
$$

with $-\frac{n}{2 k}+\frac{1}{2}>0$. Next, applying Lemma 5.4, we obtain

$$
\begin{aligned}
\|\widetilde{Q}[v, w](t)\|_{p} & \leq C \int_{0}^{t}(t-s)^{-\frac{n}{2}\left(\frac{1}{r}-\frac{1}{p}\right)-\frac{1}{2}}\|v(s)\|_{q}\|w(s)\|_{q} \mathrm{~d} s \\
& \leq C\|v\| \mathscr{X}\|w\| \mathscr{X} \int_{0}^{t}(t-s)^{-\frac{n}{2}\left(\frac{1}{r}-\frac{1}{p}\right)-\frac{1}{2}} s^{-n\left(\frac{1}{p}-\frac{1}{q}\right)} \mathrm{d} s .
\end{aligned}
$$

Notice that the integral on the right-hand side is convergent because the exponents satisfy the inequalities

$$
\begin{aligned}
-\frac{n}{2}\left(\frac{1}{r}-\frac{1}{p}\right)-\frac{1}{2}= & -1+\frac{n}{2}\left(\frac{1}{p}-\frac{1}{q}\right)>-1 \\
& -n\left(\frac{1}{p}-\frac{1}{q}\right)>-n\left(\frac{2}{q}-\frac{1}{q}\right)=-\frac{n}{q}>-1
\end{aligned}
$$

resulting immediately from the properties of the parameters $p, q, r$. Therefore,

$$
\begin{aligned}
\|\widetilde{Q}[v, w](t)\|_{p} \leq & C B\left(1-n\left(\frac{1}{p}-\frac{1}{q}\right), \frac{1}{2}-\frac{n}{2}\left(\frac{1}{r}-\frac{1}{p}\right)\right) \\
& \times t^{-\frac{n}{2}\left(\frac{1}{r}-\frac{1}{p}\right)-\frac{1}{2}-n\left(\frac{1}{p}-\frac{1}{q}\right)+1}\|v\| \mathscr{X}\|w\| \mathscr{X},
\end{aligned}
$$

where $B=B(x, y)$ denotes the beta function. Moreover, by the assumption on $r$, we have

$$
\begin{aligned}
-\frac{n}{2}\left(\frac{1}{r}-\frac{1}{p}\right)-\frac{1}{2}-n\left(\frac{1}{p}-\frac{1}{q}\right)+1 & =-\frac{n}{2 r}-\frac{n}{2 p}+\frac{n}{q}+\frac{1}{2} \\
& \leq-\frac{n}{2}\left(\frac{2}{q}+\frac{1}{n}-\frac{1}{p}\right)-\frac{n}{2 p}+\frac{n}{q}+\frac{1}{2}=0 .
\end{aligned}
$$

Consequently, inequalities (5.6) and (5.5) provide the estimate

$$
\begin{aligned}
\sup _{t>0}\|\widetilde{Q}[v, w](t)\|_{p} & \leq C \sup _{t>0}\left(\min \left\{t^{-\frac{n}{2 k}+\frac{1}{2}}, t^{-\frac{n}{2}\left(\frac{1}{r}-\frac{1}{p}\right)-\frac{1}{2}-n\left(\frac{1}{p}-\frac{1}{q}\right)+1}\right\}\right)\|v\|_{\mathscr{X}}\|w\|_{\mathscr{X}} \\
& \leq C\|v\|_{\mathscr{X}}\|w\|_{\mathscr{X}}
\end{aligned}
$$

with a positive constant $C>0$.

We proceed with the $L^{q}$-component of the norm in $\mathscr{X}$ analogously. By Lemma 5.2,

$$
t^{\frac{n}{2}\left(\frac{1}{p}-\frac{1}{q}\right)}\|\widetilde{Q}[v, w](t)\|_{q} \leq C t^{\frac{n}{2 p}-\frac{n}{2}\left(\frac{1}{q}+\frac{1}{k}\right)+\frac{1}{2}}\|u\|_{\mathscr{X}}\|v\|_{\mathscr{X}}
$$


with $\frac{n}{2 p}-\frac{n}{2}\left(\frac{1}{q}+\frac{1}{k}\right)+\frac{1}{2} \geq 0$ and, by Lemma 5.4,

$$
\begin{aligned}
t^{\frac{n}{2}\left(\frac{1}{p}-\frac{1}{q}\right)}\|\widetilde{Q}[v, w](t)\|_{q} & \leq C t^{\frac{n}{2}\left(\frac{1}{p}-\frac{1}{q}\right)}\|v\|_{\mathscr{X}}\|w\|_{\mathscr{X}} \int_{0}^{t}(t-s)^{-\frac{n}{2}\left(\frac{1}{r}-\frac{1}{q}\right)-\frac{1}{2}} s^{-n\left(\frac{1}{p}-\frac{1}{q}\right)} \mathrm{d} s \\
& =C t^{-\frac{n}{2}\left(\frac{1}{r}-\frac{1}{q}\right)-\frac{1}{2}-\frac{n}{2}\left(\frac{1}{p}-\frac{1}{q}\right)+1}\|v\|_{\mathscr{X}}\|w\|_{\mathscr{X}}
\end{aligned}
$$

with

$$
-\frac{n}{2}\left(\frac{1}{r}-\frac{1}{q}\right)-\frac{1}{2}-\frac{n}{2}\left(\frac{1}{p}-\frac{1}{q}\right)+1 \leq 0 .
$$

Therefore, there exists a constant $C>0$ independent of $t>0$ such that

$$
\begin{aligned}
& \sup _{t>0} t^{\frac{n}{2}\left(\frac{1}{p}-\frac{1}{q}\right)}\|\widetilde{Q}[v, w](t)\|_{q} \\
& \quad \leq C \sup _{t>0}\left(\min \left\{t^{\frac{n}{2 p}-\frac{n}{2}\left(\frac{1}{q}+\frac{1}{k}\right)+\frac{1}{2}}, t^{-\frac{n}{2}\left(\frac{1}{r}-\frac{1}{q}\right)-\frac{1}{2}-\frac{n}{2}\left(\frac{1}{p}-\frac{1}{q}\right)+1}\right\}\right)\|v\| \mathscr{X}\|w\| \mathscr{X} \\
& \leq C\|v\| \mathscr{X}\|w\| \mathscr{X} .
\end{aligned}
$$

Finally, it follows from inequalities (5.7) and (5.8) that

$$
\|\widetilde{Q}[v, w]\|_{\mathscr{X}} \leq \eta\|v\|_{\mathscr{X}}\|w\|_{\mathscr{X}}
$$

for a positive number $\eta$ independent of $t, v$ and $w$. Hence, if $\left\|v_{0}\right\|_{p}$ is sufficiently small, by inequality (5.4) and Proposition 3.10, there exists a mild solution of problem (5.1) in the space $\mathscr{X}$. This solution is unique by Proposition 5.1.

\subsection{Instability for $A>1$}

In this section, we prove that the constant solution $u=A$ of problem (2.2) is unstable in $L^{p}\left(\mathbb{R}^{n}\right)$ if $A>1$. Here by instability in the Lyapunov sense we mean that there exists $\varepsilon>0$ such that for all $\delta \in(0,1]$ there exists $v_{0} \in L^{p}\left(\mathbb{R}^{n}\right),\left\|v_{0}\right\|_{p}=1$ and $T>0$ such that the solution $v(t)$ corresponding to the initial condition $\delta v_{0}$ satisfies $\|v(T)\|_{p}>\varepsilon$. We modify the classical approach, which was used e.g. in $[8,20]$.

Proof of Theorem 2.4. We begin with arbitrary $\delta \in(0,1)$ and arbitrary $v_{0} \in L^{p}\left(\mathbb{R}^{n}\right)$ with $\left\|v_{0}\right\|_{p}=1$ to be chosen later on. Under the assumption on $p$, by Corollary 2.2, there exists a unique local-in-time mild solution $v \in C\left(\left[0, T_{\max }\right) ; L^{p}\left(\mathbb{R}^{n}\right)\right)$ to problem (5.1), with the initial datum $\delta v_{0}$. Suppose that this solution is global-in-time and for $a=(\sqrt{A}-1)^{2}$ define two numbers

$$
T=\sup \left\{t:\left\|v(\tau)-S_{A}(\tau) \delta v_{0}\right\|_{p} \leq \frac{\delta}{2} e^{a \tau} \text { for all } \tau \in[0, t]\right\}
$$

and $T^{\prime}=\frac{1}{a} \log \left(\frac{2}{\delta}\right)$, hence $\delta e^{a T^{\prime}}=2$.

If either $T>T^{\prime}$ or $T=\infty$, then the zero solution is unstable. Indeed, by Lemma 4.12 , for each $\gamma>0$ we may choose $v_{0} \in L^{p}\left(\mathbb{R}^{n}\right)$ with $\left\|v_{0}\right\|_{p}=1$ such that

$$
\left\|S_{A}\left(T^{\prime}\right) \delta v_{0}-e^{a T^{\prime}} \delta v_{0}\right\|_{p} \leq \gamma \delta\left\|v_{0}\right\|_{p}=\gamma \delta .
$$


By the definition of $T$ and by inequality (5.10), we obtain $\left\|v\left(T^{\prime}\right)\right\|_{p} \geq\left\|S_{A}\left(T^{\prime}\right) \delta v_{0}\right\|_{p}-\frac{\delta}{2} e^{a T^{\prime}} \geq\left\|e^{a T^{\prime}} \delta v_{0}\right\|_{p}-\gamma \delta-\frac{\delta}{2} e^{a T^{\prime}}=\frac{\delta}{2} e^{a T^{\prime}}-\gamma \delta \geq 1-\gamma$. In particular, $\left\|v\left(T^{\prime}\right)\right\|_{p} \geq \frac{1}{2}$ for $\gamma=\frac{1}{2}$.

Next, suppose that $T \leq T^{\prime}$ and consider the mild representation of the solution of problem (5.1) with the initial condition $\delta v_{0}$

$$
v(t)-S_{A}(t) \delta v_{0}=\int_{0}^{t} \nabla S_{A}(t-\tau) \cdot(v(\tau) \nabla K * v(\tau)) \mathrm{d} \tau .
$$

Lemma 5.2 with $C_{1}=\frac{3}{2} a$, estimates (4.9) and definition of $T$ in (5.9) lead to the inequality

$$
\begin{aligned}
& \left\|v(t)-S_{A}(t) \delta v_{0}\right\|_{p} \\
& \leq C \int_{0}^{t}(t-\tau)^{-\frac{n}{2} \frac{1}{q}-\frac{1}{2}} e^{\frac{3}{2} a(t-\tau)}\|v(\tau)\|_{p}^{2} \mathrm{~d} \tau \\
& \leq C \int_{0}^{t}(t-\tau)^{-\frac{n}{2} \frac{1}{q}-\frac{1}{2}} e^{\frac{3}{2} a(t-\tau)}\left(\left\|S_{A}(\tau) \delta v_{0}-v(\tau)\right\|_{p}^{2}+\left\|S_{A}(\tau) \delta v_{0}\right\|_{p}^{2}\right) \mathrm{d} \tau \\
& \leq C \int_{0}^{t}(t-\tau)^{-\frac{n}{2} \frac{1}{q}-\frac{1}{2}} e^{\frac{3}{2} a(t-\tau)}\left(\frac{\delta^{2}}{4} e^{2 a \tau}+4 \delta^{2} e^{2 a \tau}\right) \mathrm{d} \tau \\
& \leq C \delta^{2} e^{\frac{3}{2} a t} \int_{0}^{t}(t-\tau)^{-\frac{n}{2} \frac{1}{q}-\frac{1}{2}} e^{\frac{1}{2} a \tau} \mathrm{d} \tau \\
& \leq C \delta^{2} e^{2 a t} \int_{0}^{t}(t-\tau)^{-\frac{n}{2} \frac{1}{q}-\frac{1}{2}} \mathrm{~d} \tau \\
& \leq C \delta^{2} e^{2 a t} \int_{0}^{T}(T-\tau)^{-\frac{n}{2} \frac{1}{q}-\frac{1}{2}} \mathrm{~d} \tau \\
& \leq C \delta^{2} e^{2 a t}
\end{aligned}
$$

for all $t \in[0, T]$. Thus, from the definition of the number $T$ and from inequality (5.11) for $t=T$, we have the relations

$$
\frac{\delta}{2} e^{a T}=\left\|v(T)-S_{A}(T) \delta v_{0}\right\|_{p} \leq C \delta^{2} e^{2 a T}
$$

which imply the inequality $\frac{1}{2 C} \leq \delta e^{a T}$. In particular, the number $T^{*}$ defined by the equation $\delta e^{a T^{*}}=\frac{1}{2 C}$ satisfies $T^{*} \leq T$. Hence, by inequality (5.11) with $t=T^{*}$ we have

$$
\left\|v\left(T^{*}\right)\right\|_{p} \geq\left\|S_{A}\left(T^{*}\right) \delta v_{0}\right\|_{p}-\frac{1}{2} \delta e^{a T^{*}}=\left\|S_{A}\left(T^{*}\right) \delta v_{0}\right\|_{p}-\frac{1}{4 C} .
$$

Finally, we apply Lemma 4.12 with $\gamma=\frac{1}{4 k_{0} C} \leq 1$ for some fixed $k_{0} \gg 1$ and $T^{\prime}$ (recall that $\left.T^{*} \leq T \leq T^{\prime}\right)$ in order to obtain $v_{0} \in L^{p}\left(\mathbb{R}^{n}\right)$ with $\left\|v_{0}\right\|_{p}=1$ such that $\left\|v\left(T^{*}\right)\right\|_{p} \geq\left\|S_{A}\left(T^{*}\right) \delta v_{0}\right\|_{p}-\frac{1}{4 C} \geq \delta e^{a T^{*}}-\gamma \delta-\frac{1}{4 C} \geq \frac{1}{4 C}-\gamma=\frac{1}{4 C}\left(1-\frac{1}{k_{0}}\right)$. 
The proof of instability is completed because the right-hand side is independent of $\delta$.

\section{Acknowledgements}

S. Cygan and H. Wakui were supported by the Polish NCN grant 2016/23/B/ST1/00434. H. Wakui was also supported by JSPS Grant-in-Aid for JSPS Fellows Grant number JP20J00940.

Open Access. This article is licensed under a Creative Commons Attribution 4.0 International License, which permits use, sharing, adaptation, distribution and reproduction in any medium or format, as long as you give appropriate credit to the original author(s) and the source, provide a link to the Creative Commons licence, and indicate if changes were made. The images or other third party material in this article are included in the article's Creative Commons licence, unless indicated otherwise in a credit line to the material. If material is not included in the article's Creative Commons licence and your intended use is not permitted by statutory regulation or exceeds the permitted use, you will need to obtain permission directly from the copyright holder. To view a copy of this licence, visit http://creativecommons.org/licenses/ by/4.0/.

Publisher's Note Springer Nature remains neutral with regard to jurisdictional claims in published maps and institutional affiliations.

\section{REFERENCES}

[1] Adams, D.R., Hedberg, L.I.: Function spaces and potential theory, Grundlehren der Mathematischen Wissenschaften [Fundamental Principles of Mathematical Sciences], vol. 314. Springer, Berlin (1996)

[2] Arrieta, J.M., Rodriguez-Bernal, A., Cholewa, J.W., Dłotko, T.: Linear parabolic equations in locally uniform spaces. Math. Models Methods Appl. Sci. 14(2), 253-293 (2004)

[3] Bellomo, N., Bellouquid, A., Tao, Y., Winkler, M.: Toward a mathematical theory of Keller-Segel models of pattern formation in biological tissues. Math. Models Methods Appl. Sci. 25(9), 16631763 (2015)

[4] Biler, P., Cieślak, T., Karch, G., Zienkiewicz, J.: Local criteria for blowup in two-dimensional chemotaxis models. Discrete Contin. Dyn. Syst. 37(4), 1841-1856 (2017)

[5] Biler, P., Guerra, I., Karch, G.: Large global-in-time solutions of the parabolic-parabolic KellerSegel system on the plane. Commun. Pure Appl. Anal. 14(6), 2117-2126 (2015)

[6] Corrias, L., Perthame, B., Zaag, H.: Global solutions of some chemotaxis and angiogenesis systems in high space dimensions. Milan J. Math. 72, 1-28 (2004)

[7] Engel, K.J., Nagel, R.: One-parameter semigroups for linear evolution equations, Graduate Texts in Mathematics, vol. 194. Springer-Verlag, New York (2000). With contributions by S. Brendle, M. Campiti, T. Hahn, G. Metafune, G. Nickel, D. Pallara, C. Perazzoli, A. Rhandi, S. Romanelli and R. Schnaubelt

[8] Friedlander, S., Pavlović, N., Shvydkoy, R.: Nonlinear instability for the Navier-Stokes equations. Comm. Math. Phys. 264(1), 335-347 (2006)

[9] Friedman, A.: Partial Differential Equations of Parabolic Type. Prentice-Hall, Inc., Englewood Cliffs, N.J. (1964)

[10] Guo, Y., Hwang, H.J.: Pattern formation (I): the Keller-Segel model. J. Differential Equations 249(7), 1519-1530 (2010)

[11] Hardy, M.: Combinatorics of partial derivatives. Electron. J. Combin. 13(1) (2006)

[12] Horstmann, D.: From 1970 until present: the Keller-Segel model in chemotaxis and its consequences. I. Jahresber. Deutsch. Math.-Verein. 105(3), 103-165 (2003) 
[13] Karch, G., Suzuki, K.: Spikes and diffusion waves in one-dimensional model of chemotaxis. Nonlinearity 23(12), 3119-3137 (2010)

[14] Karch, G., Suzuki, K.: Blow-up versus global existence of solutions to aggregation equations. Appl. Math. (Warsaw) 38(3), 243-258 (2011)

[15] Keller, E.F., Segel, L.A.: Initiation of slime mold aggregation viewed as an instability. J. Theoret. Biol. 26(3), 399-415 (1970)

[16] Kozono, H., Sugiyama, Y.: Local existence and finite time blow-up of solutions in the 2-d KellerSegel system. J. Evol. Equ. 8(2), 353-378 (2008)

[17] Kozono, H., Sugiyama, Y., Yahagi, Y.: Existence and uniqueness theorem on weak solutions to the parabolic-elliptic Keller-Segel system. J. Differential Equations 253(7), 2295-2313 (2012)

[18] Maekawa, Y., Terasawa, Y.: The Navier-Stokes equations with initial data in uniformly local $L^{p}$ spaces. Differential Integral Equations 19(4), 369-400 (2006)

[19] Raczyński, A.: Diffusion-dominated asymptotics of solution to chemotaxis model. J. Evol. Equ. 11(3), 509-529 (2011)

[20] Shatah, J., Strauss, W.: Spectral condition for instability. In: Nonlinear PDE's, dynamics and continuum physics (South Hadley, MA, 1998), Contemp. Math., vol. 255, pp. 189-198. Amer. Math. Soc., Providence, RI (2000)

[21] Stein, E.M.: Singular integrals and differentiability properties of functions. No. 30 in Princeton Mathematical Series. Princeton University Press, Princeton (1970)

[22] Suguro, T.: Well-posedness and unconditional uniqueness of mild solutions to the Keller-Segel system in uniformly local spaces. J. Evol. Equ (2021), pp. 1-20, Published online

[23] Winkler, M.: How unstable is spatial homogeneity in Keller-Segel systems? a new critical mass phenomenon in two- and higher-dimensional parabolic-elliptic cases. Math. Ann. 373(12), 1237$1282(2019)$

[24] Wong, M.W.: Spectra of pseudodifferential operators on $L^{p}\left(\mathbb{R}^{n}\right)$. Comm. Partial Differential Equations 4(12), 1389-1401 (1979)

[25] Yagi, A.: Abstract parabolic evolution equations and their applications. Springer Monographs in Mathematics. Springer-Verlag, Berlin (2010)

\author{
Szymon Cygan, Grzegorz Karch and Krzysztof Krawczyk \\ Instytut Matematyczny \\ Uniwersytet Wrocławski \\ pl. Grunwaldzki 2/4 \\ 50-384 Wroctaw \\ Poland \\ E-mail: szymon.cygan2@uwr.edu.pl \\ Grzegorz Karch \\ E-mail: grzegorz.karch@uwr.edu.pl \\ Krzysztof Krawczyk \\ E-mail: krzysztof.krawczyk@uwr.edu.pl \\ Hiroshi Wakui \\ Faculty of Science Division I \\ Tokyo University of Science \\ 1-3 Kagurazaka \\ Shinjuku-ku Tokyo162-8601 \\ Japan \\ E-mail:hiroshi.wakui@rs.tus.ac.jp
}

Accepted: 9 July 2021 